\title{
Concurrent Use of Hypnotic Drugs and Chinese Herbal Medicine Therapies among Taiwanese Adults with Insomnia Symptoms: A Population-Based Study
}

\author{
Kuei-Hua Lee, ${ }^{1,2}$ Yueh-Ting Tsai, ${ }^{1}$ Jung-Nien Lai, ${ }^{1,3}$ and Shun-Ku Lin ${ }^{4}$ \\ ${ }^{1}$ Institute of Traditional Medicine, School of Medicine, National Yang-Ming University, Linong Road, Taipei 112, Taiwan \\ ${ }^{2}$ Department of Pharmacy, Taipei City Hospital, Songde Branch, Taipei 110, Taiwan \\ ${ }^{3}$ Department of Chinese Medicine, Taipei City Hospital, Yangming Branch, Taipei 111, Taiwan \\ ${ }^{4}$ Department of Chinese Medicine, Taipei City Hospital, Renai Branch, Taipei 106, Taiwan
}

Correspondence should be addressed to Jung-Nien Lai; ericlaill1@gmail.com

Received 12 June 2013; Revised 5 August 2013; Accepted 19 August 2013

Academic Editor: Lixing Lao

Copyright (C) 2013 Kuei-Hua Lee et al. This is an open access article distributed under the Creative Commons Attribution License, which permits unrestricted use, distribution, and reproduction in any medium, provided the original work is properly cited.

\begin{abstract}
Background. The increased practice of traditional Chinese medicine (TCM) worldwide has raised concerns regarding herb-drug interactions. The purpose of our study is to analyze the concurrent use of Chinese herbal products (CHPs) among Taiwanese insomnia patients taking hypnotic drugs. Methods. The usage, frequency of services, and CHP prescribed among 53,949 insomnia sufferers were evaluated from a random sample of 1 million beneficiaries in the National Health Insurance Research Database. A logistic regression method was used to identify the factors that were associated with the coprescription of a CHP and a hypnotic drug. Cox proportional hazards regressions were performed to calculate the hazard ratios (HRs) of hip fracture between the two groups. Results. More than 1 of every 3 hypnotic users also used a CHP concurrently. Jia-Wei-Xiao-Yao-San (Augmented Rambling Powder) and Suan-Zao-Ren-Tang (Zizyphus Combination) were the 2 most commonly used CHPs that were coadministered with hypnotic drugs. The HR of hip fracture for hypnotic-drug users who used a CHP concurrently was 0.57 -fold $(95 \% \mathrm{CI}=0.47-0.69)$ that of hypnotic-drug users who did not use a CHP. Conclusion. Exploring potential CHP-drug interactions and integrating both healthcare approaches might be beneficial for the overall health and quality of life of insomnia sufferers.
\end{abstract}

\section{Introduction}

Inadequate sleep and sleep disorders are common, and are associated with an increased risk of poor health, diminished work and academic performance, and negative safety outcomes that have critical clinical and economic ramifications [1]. Although substantial progress has recently been made in the pharmacologic treatment of insomnia, including the use of benzodiazepines and Z-drugs (zaleplon, zolpidem, and zopiclone), adverse effects and the potential for abuse, addiction, and the development of tolerance have led to poor compliance for hypnotic regimens among insomniac patients [2,3]. Many poor sleepers have turned to traditional Chinese medicine (TCM) remedies to manage their symptoms because they believe that such treatments exert fewer subjective residual effects [4].
Although TCM remedies are promoted as natural, and therefore harmless, complementary, and alternative medicines that can also be used in Western countries $[5,6]$, data are limited regarding their safety when used in combination with hypnotic drugs, particularly regarding the risk of herb-drug interactions, such as that resulting from interference with the clearance of either of the drugs. Studies on the prevalence of hypnotic-drug use and the prescription patterns of TCM remedies among hypnotic-drug users are scant.

Comprising unique traditional therapies for various ailments, TCM has been used in Taiwan for hundreds of years, and its popularity remains unabated, despite the present availability of modern medical care in Taiwan. In addition, one distinguishing feature of the national healthcare system in Taiwan is the coexistence of modern Western medicine (WM) and TCM, including acupuncture and manipulative 
therapies and Chinese herbal products (CHPs), claims for which have been covered by the National Health Insurance (NHI) system since 1995 [7].

People in Taiwan are free to choose from care offered by WM clinics or TCM clinics. With an insured rate of $98 \%$ to $99 \%$, the random sample that comprises the NHI research database (NHIRD) is representative of the general population of Taiwan and should allow a reasonably accurate assessment of the concurrent usage of TCM and modern medical resources in Taiwan; therefore, the NHIRD provides an ideal platform for pharmacoepidemiological studies [8]. Our study aimed to describe the demographics and patterns of CHP usage among hypnotic-drug users and, by using a large population-based retrospective database, explore the risk of hip fracture among a cohort of hypnotic-drug users prescribed with a CHP concurrently compared with hypnotic-drug users who did not use Chinese herbs. Our findings provide evidence-based information for formulating appropriate management strategies for drug safety and integrative medicine.

\section{Materials and Methods}

2.1. Data Source and Participants. Our study protocols were approved by the Institutional Review Board of the Committee on Chinese Medicine and Pharmacy (CCMP), Department of Health, Taiwan. Our population-based study retrospectively analyzed the reimbursement records of 1 million NHI beneficiaries in the NHIRD that had been previously selected at random from the 22 million beneficiaries of the NHI to determine the prevalence of concurrent CHP and hypnotic use between January 1, 2002, and December 31, 2008, in Taiwan. The electronic records of the NHIRD use beneficiary identification numbers that are encrypted and maintained by the National Health Research Institutes (NHRI) of Taiwan $[9,10]$.

The NHIRD records contain demographic information, including age and sex, and clinical data, including all records of clinical visits and hospitalizations, and all information regarding prescribed drugs and dosages, including those for CHPs. The diagnoses used in the NHIRD are coded according to the International Classification of Diseases, Ninth Revision, Clinical Modification (ICD-9-CM) [11].

Because the cost of all prescribed hypnotic drugs is reimbursed by the NHI, they cannot be dispensed at a pharmacy without a physician's prescription. To construct a fixed cohort, all outpatients with insomnia who used hypnotic drugs, including benzodiazepines, zolpidem, and zopiclone, were reviewed. For the purpose of studying the use of CHPs, we downloaded the claims forms for reimbursed CHPs from the website of the Bureau of National Health Insurance. The corresponding information regarding the CHP was then obtained from the CCMP website, including the name of each herb, the proportion of each constituent of the mixture, the date and period of approval for the drug, the CCMP manufacturer code, and the name of the CHP manufacturer. All CHPs with the same CCMP standard formula are classified in the same category, regardless of slight variations in the products among different CHP manufacturers [12]. For our analysis of the demographic and clinical variables, a coprescription of a
CHP and a hypnotic drug (defined as prescriptions for both issued simultaneously or issued separately with overlapping treatment periods) was used to determine the use of a CHP and a hypnotic drug on the same day.

The participant selection from the NHIRD was performed as shown in Figure 1. We reviewed all beneficiaries with at least three outpatient visits with any nonorganic insomnia (ICD-9-CM codes 307.40-42, 307.45-49, 780.50-52, and 780.55-59) and excluded patients who were admitted to hospital and received a hip fracture diagnosis between 1999 and $2001(n=138)$. Patients with hyperinsomnia (ICD-9-CM codes 327, 307.43, 307.44, 780.53, and 780.54) for the calendar year in which it occurred were excluded. The prevalent insomnia cases diagnosed before the end of 2001 $(n=9,242)$ and patients under 20 years of age $(n=4,691)$ were excluded to ensure that all participants had been newly diagnosed with adult insomnia. Patients with incomplete data for age or sex $(n=21)$ and insomniac patients not taking hypnotic drugs $(n=10,628)$ were also excluded.

2.2. Study Variables. To identify the key factors associated with the coadministration of hypnotics and CHP among insomnia sufferers (CAHCHP as an acronym for this population), we selected the demographic factors according to previous studies $[1,7,8]$. Patients were classified, based on age, into one of seven groups as follows: 20-29 years, 30-39 years, 40-49 years, 50-59 years, 60-69 years, 7079 years, and $\geq 80$ years. The geographic areas of Taiwan in which patients resided were classified as one of the following seven regions: Taipei city, Kaohsiung city, Northern region, Central region, Southern region, Eastern region, and Outlying islands. Patients' monthly income in New Taiwan Dollars (NT\$) was categorized as one of the following four levels: \$0, \$1-\$19,999, \$20,000-\$39,999, and $\geq \$ 40,000$.

The variables for hypnotic-drug use included in our analyses were defined according to the specific proprietary hypnotic preparation used during the study period. We categorized the types of preparation used as follows: long-half-life benzodiazepine; short-half-life benzodiazepine; zolpidem; zopiclone; and mixed regimens, including two, three, or more of the aforementioned preparations.

To estimate the impact of CHP use on the rate of hip fracture, we selected subjects who were first recorded with a diagnosis of hip fracture (ICD-9-CM codes 820.0-820.9) between January 1, 2002, and December 31, 2008. Furthermore, we also analyzed the risk of hip fracture according to the period of time patients were administered hypnotic drugs ( $\geq 30$ days and $<30$ days).

2.3. Statistical Analysis. Data analysis was conducted using descriptive statistics, including the prescription rates of patients' concurrent use of a CHP and a sedative hypnotic drug stratified by age and sex, indications for the prescribed $\mathrm{CHP}$, and the most frequently coprescribed herbal formulas for treating insomnia. The indications were coded according to the ICD-9-CM and grouped into different broader disease categories. The ICD-9-CM codes 460-519 were classified as diseases of the respiratory system. Codes 780-799 were grouped as symptoms, signs, and ill-defined conditions, and 


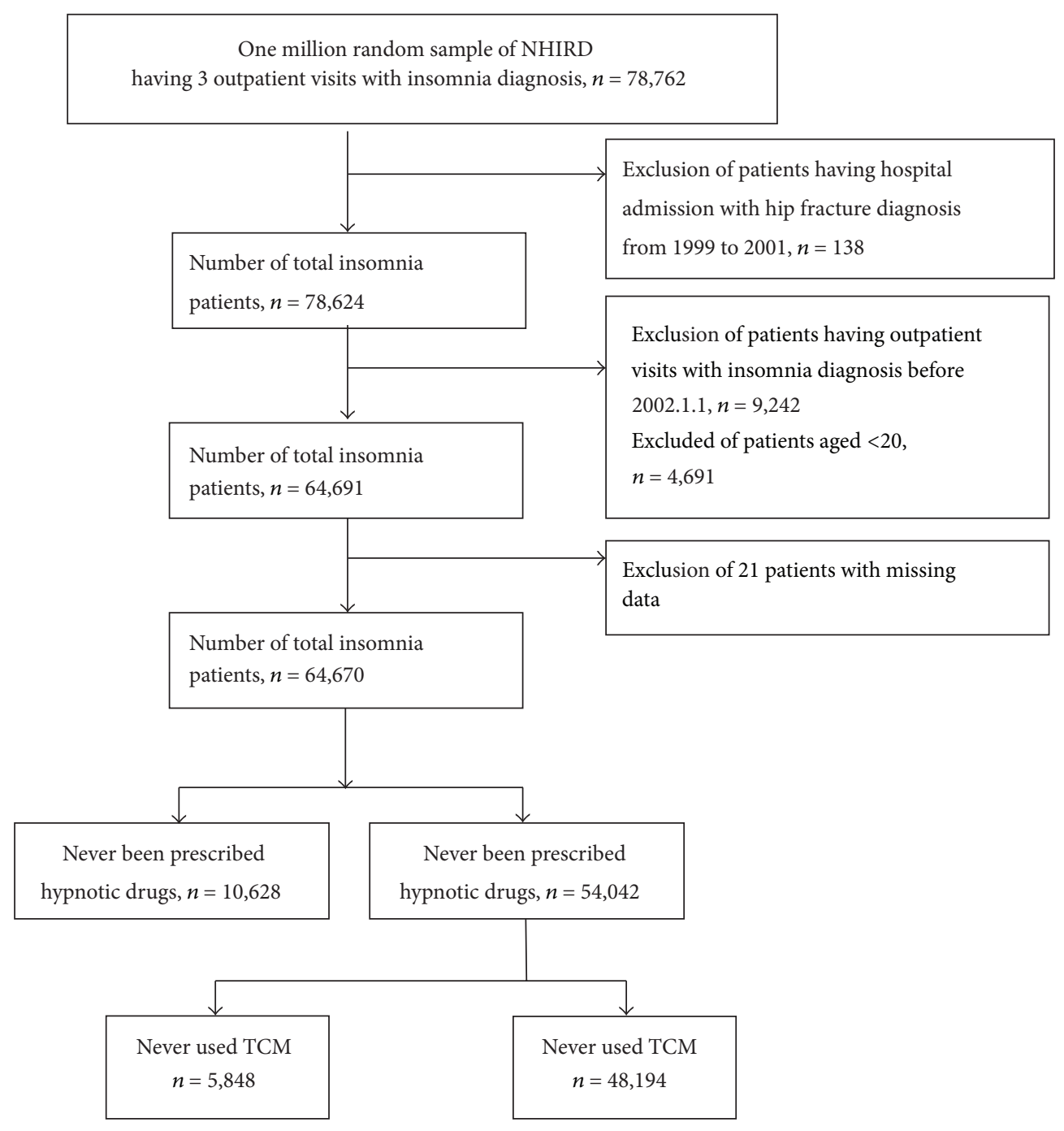

FIGURE 1: Flowchart of recruitment of subjects with insomnia from the 1 million random sample of the National Health Insurance Research Database (NHIRD) from 2002 to 2008 in Taiwan.

codes 520-579 were classified as diseases of the digestive system. Multiple logistic regression was conducted to evaluate factors that correlated with CHP use based on the odds ratio (OR) and the 95\% confidence interval (CI). Cox proportional hazards regressions were performed to calculate the hazard ratio (HR) of hip fracture among hypnotic-drug users who used a CHP concurrently and hypnotic-drug users who did not use Chinese herbs. A significance level of $\alpha=0.05$ was selected. The SAS statistical software, version 9.3 (SAS Institute, Cary, NC, USA), was used for data management and analyses.

\section{Results}

The database of outpatient claims contained information for 64,670 sleep-disturbance sufferers during the 2002-2008 period. A total of 54,042 (83.6\%) insomnia patients treated with sedative-hypnotic drugs were included in our study. Most of these patients used short-acting benzodiazepines. Most of the sedative-hypnotic users (89.2\%) also sought care from TCM practitioners. Up to $37.0 \%(n=19,994)$ of the sedative-hypnotic users received a coprescription for a CHP, resulting in the coadministration of a sedative-hypnotic drug with a CHP. Such patients who were coadministered the two types of drug are hereafter referred to as CAHCHP patients. A total of 19,994 CAHCHP patients were identified in our insomnia cohort.

The hypnotic users who did not use a CHP were significantly older and more likely to be male than were the CAHCHP patients. More CAHCHP patients had income levels of $\$ 20,000$ to $\$ 39,999$ and above, resided in Central Taiwan, and used two or more types of sedative-hypnotic drugs than the sedative-hypnotic users who did not use a CHP. The adjusted ORs (aORs) and 95\% CIs calculated using the logistic regression model are displayed in Table 1. After adjusting for other factors, the aORs of CAHCHP patients $(\mathrm{aORs}=2.49)$ were higher for women than for men $(\mathrm{aORs}=$ $1.00)$ except patients aged 80 years and older. Compared with the patients aged $40-49$ years $(\mathrm{aORs}=1.00)$, the aORs of the CAHCHP patients decreased with age. Patients who used at 
TABLE 1: Demographic characteristics and results of multiple logistic regression showing the adjusted odds ratio (aOR) and 95\% confidence interval (CI) of patients with newly diagnosed insomnia ever been prescribed hypnotic drugs from the 1 million random sample of the National Health Insurance Research Database (NHIRD) from 2002 to 2008 in Taiwan.

\begin{tabular}{|c|c|c|c|c|c|}
\hline \multirow[t]{2}{*}{ Characteristics } & \multirow[t]{2}{*}{ Total no. } & \multirow{2}{*}{$\begin{array}{l}\text { Hypnotic users } \\
\text { without using } \\
\text { Chinese medicine, } \\
\text { no. }(\%)\end{array}$} & \multirow{2}{*}{$\begin{array}{l}\text { Hypnotic users } \\
\text { coprescribed } \\
\text { Chinese medicine, } \\
\text { no. }(\%)\end{array}$} & \multicolumn{2}{|c|}{$\begin{array}{l}\text { Hypnotic users coprescribed } \\
\text { Chinese medicine/hypnotic users } \\
\text { without using Chinese medicine }\end{array}$} \\
\hline & & & & Odds ratio & $(95 \% \mathrm{CI})$ \\
\hline $\begin{array}{l}\text { Numbers of hypnotic users among insomnia } \\
\text { population }\end{array}$ & 54,042 & $5,848(10.8)$ & $19,994(37.0)$ & & \\
\hline \multicolumn{6}{|l|}{ Sex } \\
\hline Male & 19,354 & $3,234(55.3)$ & $6,350(31.8)$ & 1.00 & \multirow{2}{*}{$(2.34-2.66)$} \\
\hline Female & 34,688 & $2,614(44.7)$ & $13,644(68.2)$ & 2.49 & \\
\hline Mean (SD) age at inclusion, years & $48.8(15.4)$ & $55.1(17.2)$ & $49.9(14.9)$ & & \\
\hline \multicolumn{6}{|l|}{ Age groups at inclusion, years no. (\%) } \\
\hline $20 \sim 29$ & 5,616 & $362(6.2)$ & $1,631(8.2)$ & 1.06 & $(0.93-1.22)$ \\
\hline Male & & $184(3.0)$ & $477(5.8)$ & & \\
\hline Female & & $178(3.2)$ & $1,154(2.4)$ & & \\
\hline 30 39 & 11,105 & $891(15.2)$ & $3,820(19.1)$ & 1.03 & $(0.94-1.14)$ \\
\hline Male & & $506(6.6)$ & $1,184(13.2)$ & & \\
\hline Female & & $385(8.6)$ & $2,636(5.9)$ & & \\
\hline $40 \sim 49$ & 13,531 & $1,193(20.4)$ & $5,074(25.4)$ & 1.00 & \\
\hline Male & & $675(8.9)$ & $1,536(17.7)$ & & \\
\hline Female & & $518(11.5)$ & $3,538(7.7)$ & & \\
\hline $50 \sim 59$ & 10,637 & $1,072(18.3)$ & $4,184(20.9)$ & 0.85 & $(0.77-0.93)$ \\
\hline Male & & $550(8.9)$ & $1,118(15.3)$ & & \\
\hline Female & & $522(9.4)$ & $3,066(5.6)$ & & \\
\hline $60 \sim 69$ & 6,657 & $865(14.8)$ & $2,798(14.0)$ & 0.76 & $(0.68-0.84)$ \\
\hline Male & & $488(6.5)$ & $962(9.2)$ & & \\
\hline Female & & $377(8.3)$ & $1,836(4.8)$ & & \\
\hline $70 \sim 79$ & 4,787 & $922(15.8)$ & 1,951 (9.8) & 0.52 & $(0.46-0.58)$ \\
\hline Male & & $545(6.5)$ & $821(5.7)$ & & \\
\hline Female & & $377(9.3)$ & $1,130(4.1)$ & & \\
\hline Over 80 & 1,709 & $543(9.3)$ & $536(2.7)$ & 0.24 & $(0.21-0.28)$ \\
\hline Male & & $286(4.4)$ & $252(1.4)$ & & \\
\hline Female & & $257(4.9)$ & $284(1.3)$ & & \\
\hline \multicolumn{6}{|l|}{$\$ N T /$ month (Premiums) } \\
\hline 0 & 11,542 & $1,279(21.9)$ & $4,735(23.7)$ & 1.00 & \\
\hline $1-19,999$ & 27,391 & $3,268(55.9)$ & $10,123(50.6)$ & 0.85 & $(0.79-0.92)$ \\
\hline $20,000-39,999$ & 9,601 & $729(12.5)$ & $3,303(16.5)$ & 1.13 & $(1.01-1.26)$ \\
\hline$\geq 40,000$ & 5,508 & $572(9.8)$ & $1,833(9.2)$ & 1.03 & $(0.91-1.17)$ \\
\hline \multicolumn{6}{|l|}{ Insured area } \\
\hline Taipei city & 9,495 & 1,159 (19.8) & $3,239(16.2)$ & 1.00 & \\
\hline Kaohsiung city & 3,735 & $364(6.2)$ & $1,392(7.0)$ & 1.46 & $(1.27-1.68)$ \\
\hline Northern Taiwan & 14,900 & $1,848(31.6)$ & $4,929(24.7)$ & 0.98 & $(0.90-1.08)$ \\
\hline Central Taiwan & 12,554 & $824(14.1)$ & $5,490(27.5)$ & 2.49 & $(2.24-2.77)$ \\
\hline Southern Taiwan & 11,910 & $1,401(24.0)$ & $4,407(22.0)$ & 1.28 & $(1.16-1.41)$ \\
\hline Eastern Taiwan & 1,078 & $155(2.7)$ & $439(2.2)$ & 1.01 & $(0.82-1.24)$ \\
\hline Outlying islands & 370 & $97(1.7)$ & $98(0.5)$ & 0.36 & $(0.27-0.49)$ \\
\hline
\end{tabular}


TABle 1: Continued.

\begin{tabular}{|c|c|c|c|c|c|}
\hline \multirow[t]{2}{*}{ Characteristics } & \multirow[t]{2}{*}{ Total no. } & \multirow{2}{*}{$\begin{array}{l}\text { Hypnotic users } \\
\text { without using } \\
\text { Chinese medicine, } \\
\text { no. (\%) }\end{array}$} & \multirow{2}{*}{$\begin{array}{l}\text { Hypnotic users } \\
\text { coprescribed } \\
\text { Chinese medicine, } \\
\text { no. }(\%)\end{array}$} & \multicolumn{2}{|c|}{$\begin{array}{l}\text { Hypnotic users coprescribed } \\
\text { Chinese medicine/hypnotic users } \\
\text { without using Chinese medicine }\end{array}$} \\
\hline & & & & Odds ratio & $(95 \% \mathrm{CI})$ \\
\hline \multicolumn{6}{|l|}{ Types and prescription patterns of hypnotics } \\
\hline Usual treatment regimens & 16,182 & $1,352(23.1)$ & $3,310(16.6)$ & 1.00 & \\
\hline Zolpidem & 1,623 & $350(6.0)$ & $265(1.3)$ & & \\
\hline Zopiclone & 109 & $16(0.3)$ & $20(0.1)$ & & \\
\hline BZD-long & 8,657 & $293(5.0)$ & $1,703(8.5)$ & & \\
\hline BZD-short & 5,793 & $693(11.9)$ & $1,322(6.6)$ & & \\
\hline \multicolumn{6}{|l|}{ Mixed regimens } \\
\hline Co-prescribed two types of hypnotics & 18,466 & $2,168(37.1)$ & $6,734(33.6)$ & 1.30 & $(1.20-1.42)$ \\
\hline Zolpidem + Zopiclone & 79 & $20(0.3)$ & $18(0.1)$ & & \\
\hline Zolpidem + BZD-long & 1,796 & $275(4.7)$ & $486(2.4)$ & & \\
\hline Zolpidem + BZD-short & 4,418 & $849(14.5)$ & $1,499(7.5)$ & & \\
\hline Zopiclone + BZD-long & 136 & $25(0.4)$ & $40(0.2)$ & & \\
\hline Zopiclone + BZD-short & 308 & $63(1.1)$ & $99(0.5)$ & & \\
\hline BZD-long + BZD-short & 11,729 & $936(16.0)$ & $4,592(23.0)$ & & \\
\hline Coprescribed more than two types of hypnotics & 19,394 & $2,328(39.8)$ & $9,950(49.8)$ & 1.90 & $(1.75-2.06)$ \\
\hline
\end{tabular}

NT\$ refers to new Taiwan dollars, of which 1 US $\$=30$ NT\$.

least two types of sedative hypnotic drugs (two types: OR = 1.30; 95\% CI: $1.20-1.42$ and $\geq$ three types: $O R=1.90 ; 95 \% \mathrm{CI}$ : 1.75-2.06) were more likely to be CHP users than were the patients who used only one sedative hypnotic drug.

By analyzing the percentage distribution of the 19,994 CAHCHP patients, we observed that sleep disturbance $(1,450,671$ TCM visits) was the most common major disease category for coprescription events, followed by diseases of the digestive system $(1,349,092$ TCM visits) and the diseases of the respiratory system $(1,319,859$ TCM visits), as summarized in Table 2. As shown in Table 3, zolpidem, alprazolam, lorazepam, estazolam, and fludiazepam were the five sedative hypnotic drugs that were most frequently coprescribed with a CHP. The details of the most frequently used CHP formulas are shown in Table 3. Suan-Zao-Ren-Tang (Zizyphus Combination) was the most frequently used CHP, followed by JiaWei-Xiao-Yao-San (Augmented Rambling Powder) and TianWang-Bu-Xin-Dan (Ginseng and Zizyphus Combination). The average number of days of concurrent use of a sedative hypnotic drug and a CHP was approximately 30 days per CAHCHP patient during the 7-year study period.

The HRs of hip fracture for CAHCHP patients and hypnotic-drug users who did not use Chinese herbs are presented in Table 4. After adjusting for potential confounders, the HR of hip fracture for CAHCHP patient was 0.57 -fold (95\% CI $=0.47-0.69)$ that of comparison subjects. We further analyzed the risk of hip fracture according to the length of hypnotic treatment. Table 4 shows that, compared with hypnotic-drug users who did not use Chinese herbs, the HR of hip fracture for CAHCHP patients who were prescribed hypnotics for $\geq 30$ days was $0.62(95 \% \mathrm{CI}=0.50-0.77)$ and as low as 0.33 (95\% CI $=0.20-0.54)$ for CAHCHP patient who were prescribed hypnotics for $<30$ days. The incidence rate of hip fracture among the CAHCHP patients was no higher than that of hypnotic-drug users who did not use Chinese herbs ( $\geq 30$-day group: 3.4 versus 6.5 per 100 person-years; $<30$-day group: 2.9 versus 11.1 per 100 person-years).

\section{Discussion}

According to our review of the literature, this study is the first to use a random population-based cohort to document the coprescription of hypnotic drugs and CHPs in insomnia patients in Taiwan. We observed that hypnotic use is common among insomnia patients in Taiwan and that benzodiazepines were the most frequently prescribed category of sedative hypnotic, as shown in Table 1. Previous studies have reported that patients treated with benzodiazepines may be at higher risk for falls and have been associated with an increased risk of hip fracture. Both physicians and patients should be aware of the association between hypnotic use and the potential risk for injury in Taiwan. The possibility of recall or selection bias can be excluded because we included patients who were newly diagnosed with insomnia by qualified conventional physicians during the 2002-2008 period from a random sample of the population-based $\mathrm{NHI}$ database.

The prevalence of the concurrent use of sedative hypnotics and CHPs was 37\% (Figure 1). The abuse of benzodiazepines has become a serious problem in North America [13]. The fear of sedative hypnotic side effects or dependency may motivate patients to seek TCM therapies [2, 3, 14]. Although barbiturates have been reported to be the most prescribed hypnotic in Taiwan, our findings indicate a change 
TABLE 2: Frequency distribution of traditional Chinese medicine (TCM) visits by major disease categories (according to 9th ICD codes) in subjects with insomnia ever been prescribed hypnotic drugs from 2002 to 2008 in Taiwan.

\begin{tabular}{|c|c|c|c|}
\hline \multirow[b]{2}{*}{ Diagnosis } & \multirow[b]{2}{*}{ ICD-9-CM codes } & \multicolumn{2}{|c|}{ Treatment days (people) } \\
\hline & & Chinese herbal remedies & $\begin{array}{c}\text { Acupuncture and manipulative } \\
\text { therapies }\end{array}$ \\
\hline Infectious and parasitic diseases & 001-139 & $46,426(2,027)$ & $47(14)$ \\
\hline Neoplasms & $140-239$ & $63,080(904)$ & $777(32)$ \\
\hline $\begin{array}{l}\text { Endocrine, nutritional, and metabolic } \\
\text { diseases and immunity disorders }\end{array}$ & $240-279$ & $213,461(4,425)$ & $890(90)$ \\
\hline Psychotic diseases & $290-319$ & $164,603(4,417)$ & $1,781(135)$ \\
\hline Somnambulism & 307.4 & $57,639(2,073)$ & $268(34)$ \\
\hline Others & 507.4 & $106,964(2,542)$ & $1,513(105)$ \\
\hline Disease of nervous system and sense organs & $320-389$ & $332,372(10,391)$ & $6,057(1,200)$ \\
\hline Disease of circulation system & $390-459$ & $257,825(6,088)$ & $4,384(288)$ \\
\hline Disease of respiratory system & $460-519$ & $1,319,859(26,741)$ & $2,334(253)$ \\
\hline Disease of digestive system & $520-579$ & $1,349,092(25,113)$ & $3,501(275)$ \\
\hline Disease of genitourinary system & $580-629$ & $824,093(15,653)$ & $4,102(189)$ \\
\hline Disease of the skin and subcutaneous tissues & $680-709$ & $238,578(8,315)$ & $857(72)$ \\
\hline $\begin{array}{l}\text { Disease of musculoskeletal system and } \\
\text { connective tissue }\end{array}$ & $710-739$ & $647,207(17,991)$ & $135,465(23,473)$ \\
\hline Symptom, signs, and ill-defined conditions & $780-799$ & $3,036,297(40,342)$ & $11,642(1,348)$ \\
\hline Sleep disturbance & 780.5 & $1,450,671(35,386)$ & $6,733(590)$ \\
\hline Others & 700.0 & $1,585,626(29,955)$ & $4,909(845)$ \\
\hline Injury and poisoning & $800-999$ & $46,331(3,324)$ & $138,053(26,466)$ \\
\hline Supplementary classification & V01-V82, E800-E999 & $\begin{array}{c}375(30) \\
0(0)\end{array}$ & $\begin{array}{l}2(2) \\
4(1)\end{array}$ \\
\hline Others* & & $67,793(2,570)$ & $1,053(353)$ \\
\hline Total & & $8,607,392(45,847)$ & $310,943(34,454)$ \\
\hline
\end{tabular}

${ }^{*}$ Include ranges of 280-289, 630-677, 740-759, and 760-779 ICD-9-CM code and missing data.

TABLE 3: The top five coprescribed Chinese formulas and sedative and hypnotic drugs for treating insomnia (ICD9: 307.4 or 780.5 ) between 2002 and 2008.

\begin{tabular}{|c|c|c|c|}
\hline Chinese Medicine-sedative and hypnotic drugs & $\begin{array}{l}\text { Total days of } \\
\text { coprescribing }\end{array}$ & $\begin{array}{l}\text { Total people of } \\
\text { coprescribing }\end{array}$ & $\begin{array}{c}\text { Average days of } \\
\text { coprescribing Chinese } \\
\text { and Western medicine } \\
\text { (days/people) }\end{array}$ \\
\hline \multicolumn{4}{|l|}{ Total } \\
\hline Formulae & $1,507,601$ & 18,837 & \\
\hline Jia-Wei-Xiao-Yao-San (Augmented Rambling Powder) & 70,708 & 4,029 & 17.5 \\
\hline Suan-Zao-Ren-Tang (Zizyphus Combination) & 58,115 & 3,646 & 15.9 \\
\hline Tian-Wang-Bu-Xin-Dan (Ginseng and Zizyphus Combination) & 46,040 & 2,744 & 16.8 \\
\hline Chai-Hu-Jia-Long-Gu-Mu-Li-Tang (Bupleurum and Mu Li Combination) & 39,494 & 2,409 & 16.4 \\
\hline Gan-Mai-Da-Zao-Tang (Licorice and Jujube Combination) & 38,158 & 2,097 & 18.2 \\
\hline Sedative and hypnotic drugs & $2,041,718$ & 19,994 & \\
\hline Zolpidem $^{1}$ & 462,395 & 6,415 & 30.6 \\
\hline Alprazolam ${ }^{1}$ & 313,833 & 4,990 & 27.0 \\
\hline Lorazepam $^{1}$ & 276,865 & 5,053 & 24.4 \\
\hline Estazolam $^{1}$ & 180,646 & 2,400 & 31.3 \\
\hline Fludiazepam $^{1}$ & 171,842 & 2,229 & 34.0 \\
\hline
\end{tabular}

\footnotetext{
${ }^{1}$ Short-acting BZD (elimination half time $\leq 24$ hours).
} 
TABLE 4: Number (no.) of new cases, population-at-risk, and incidence rates and hazard ratios (HR); 95\% confidence intervals (CI) for hip fracture estimated from multivariate Cox regression model on a random sample of the National Health Insurance Research Database among sample subjects and followed from 2002 to 2008.

\begin{tabular}{lccc}
\hline $\begin{array}{l}\text { Presence of hip fracture during the follow-up } \\
\text { period }\end{array}$ & $\begin{array}{l}\text { Hypnotic users without } \\
\text { using Chinese medicine, } \\
\text { no. cases/population }\end{array}$ & $\begin{array}{l}\text { Hypnotic users } \\
\text { coprescribed Chinese } \\
\text { medicine, no. } \\
\text { cases/population }\end{array}$ & $\begin{array}{l}\text { Hypnotic users coprescribed } \\
\text { Chinese medicine/hypnotic users } \\
\text { without using Chinese medicine } \\
\text { (9R CI) }\end{array}$ \\
\hline $\begin{array}{l}\text { Numbers of hip fractures among hypnotic users } \\
\geq 30 \text { days }\end{array}$ & $152 / 5,848$ & $294 / 19,994$ & 0.57 \\
Incidence rate per 1,000 person-years & $123 / 5,045$ & $260 / 17,149$ & 0.62 \\
$<30$ days & 6.5 & 3.4 & $0.47-0.69)$ \\
Incidence rate per 1,000 person-years & $29 / 803$ & $34 / 2,845$ & $(0.50-0.77)$ \\
\hline
\end{tabular}

in the prescribing habits of physicians, with nonbarbiturates, particularly zolpidem, being coprescribed more frequently than barbiturates. We also observed that poor sleepers who were prescribed multiple types of hypnotics were more likely to use a CHP concurrently compared with patients who did not seek TCM treatment.

In Taiwan, the prescribing of hypnotic drugs must be accompanied by a standard ICD-9-CM diagnosis code $[9,10]$ to adhere to the requirements for NHI claims reimbursement. The change in prescription patterns that we observed might represent an attempt by physicians to achieve a greater therapeutic effect for patients with a history of tolerance, poor response, or dependence in previous hypnotic-drug treatment [15] or it might reflect an increasing concern among physicians regarding the potential abuse of sedative hypnotic drugs. Considering the increasing incidence of hypnotic$\mathrm{CHP}$ coprescriptions, we suggest that a more critical attitude toward the use of hypnotics and CHPs in combination is required among both physicians and hypnotic-drug users. We observed that hypnotic-drug users who use a CHP concurrently were not more strongly associated with an increased risk of hip fracture than were hypnotic users who did not use Chinese herbs. Little is known regarding the potential interactions of CHPs with hypnotic drugs; therefore, healthcare providers and public-health policy analysts should focus greater attention on the potential long-term impact of this particular healthcare-seeking behavior on health outcomes [16].

Our previous clinical trials demonstrated that Jia-WeiXiao-Yao-San (Augmented Rambling Powder) and SuanZao-Ren-Tang (Zizyphus Combination), the two CHPs that were most commonly coprescribed with a hypnotic drug, may be an efficacious therapy for improving sleep quality $[5,6]$, as shown in Table 3. Among the five most frequently coprescribed formulas for treating insomnia, Tian-Wang-BuXin-Dan (Ginseng and Zizyphus Combination), Gan-MaiDa-Zao-Tang (Licorice and Jujube Combination), and Chai$\mathrm{Hu}$-Jia-Long-Gu-Mu-Li-Tang (Bupleurumand $\mathrm{Mu} \mathrm{Li}$ Combination) all have a long history of use in Taiwan. They are said to nourish the blood and calm the nerves and are frequently prescribed by TCM practitioners to alleviate sleep disturbances [17].

The concomitant use of benzodiazepines and Z-drugs was the most common combination of hypnotics that were used by patients who used multiple types of hypnotics. Because of a lack of sufficient evidence supporting this less judicious prescription pattern, we suggest that such patients may be more likely to "doctor shop," resulting in the high prevalence of the concurrent use of CHPs and hypnotic drugs. Our findings indicate that the lower incidence rate and HR of hip fracture in CAHCHP patients than that in hypnotic-drug users who did not use Chinese herbs might imply that TCM physicians in Taiwan encouraged insomnia patients to coadminister CHPs and hypnotic drugs to improve sleep quality, which might allow lower hypnotic dosages and reduce the risk of hypnotic adverse effects and dependence. However, insufficient evidence exists to support such a conclusion.

Although previous studies have demonstrated that acupuncture might be an alternative therapy for insomnia [18, 19], our data indicated that insomnia patients typically sought acupuncture treatment for injury, poisoning, and diseases of the musculoskeletal system and connective tissue. Following insomnia, diseases of the digestive system were the second most frequent disease category for TCM visits by CAHCHP patients. These results indicate that health care providers should also address the general health condition of insomnia patients, particularly gastroenterological symptoms, to identify possible causes of insomnia and provide appropriate treatment for other such medical needs that do not require hypnotic treatment. Further studies are warranted to investigate the cost effectiveness of the coadministration of hypnotic drugs with CHPs and comprehensive gastroenterological care for insomnia patients, particularly those with a history of hypnotic dependence.

Our study has three limitations: first, the NHI only reimburses the cost of the CHP; the cost of decoction is not reimbursed, which may have affected patients' decisions to use CHPs. Thus, the frequency of the concurrent use of hypnotic drugs and CHPs may be underestimated in our results. However, because the NHI provides comprehensive coverage and the copayment for prescriptions is always $\$ 50$ (approximately equal to US $\$ 1.50$ ), which is less than the typical cost of herbs sold in Taiwan's markets, the likelihood that patients purchased herbs outside of the NHI system is low. Second, we were unable to draw any conclusions regarding the relationship between the severity of the insomnia and TCM usage because such clinical data are not included in the NHIRD. Third, the retrospective design of our study 
and the lack of a randomized placebo group might diminish the statistical power of our findings. Thus, our results must be interpreted cautiously because we cannot exclude the possibility of placebo effects.

\section{Conclusion}

Our findings may have implications for the treatment of insomnia patients. Our results suggest that, with equal availability of conventional medical and TCM care, more than one-third of the hypnotic-drug users used CHPs concurrently for the relief of sleep disturbance and gastroenterological symptoms. Recognizing the benefits of TCM, exploring potential interactions and adverse effects, and integrating both healthcare approaches might be beneficial to the overall health and quality of life of insomnia patients, particularly those with a history of hypnotic dependence. Thus, health care providers should proactively explore personalized, optimal detoxification for hypnotic dependence while attending to patients' psychosocial and physical needs.

\section{Acknowledgments}

This research was conducted at the Institute of Traditional Medicine at the School of Medicine, National Yang-Ming University. The authors would like to express their sincere gratitude for the support provided for this project in the form of grants from the CCMP (CCMP95-TP004) and the NHRI (NHRI-EX96-9204PP).

\section{References}

[1] 2011 National Institutes of Health Sleep Disorders Research Plan, "Research: NIH Sleep Disorders Research Plan," National Institutes of Health, 2013, http://www.nhlbi.nih.gov/health/ prof/sleep/sleep_splan.htm.

[2] P. K. Sethi and D. C. Khandelwal, "Zolpidem at supratherapeutic doses can cause drug abuse, dependence and withdrawal seizure," Journal of Association of Physicians of India, vol. 53, pp. 139-140, 2005.

[3] C. Victorri-Vigneau, E. Dailly, G. Veyrac, and P. Jolliet, "Evidence of zolpidem abuse and dependence: results of the French Centre for Evaluation and Information on Pharmacodependence (CEIP) network survey," British Journal of Clinical Pharmacology, vol. 64, no. 2, pp. 198-209, 2007.

[4] Y. Yuwen, N. N. Shi, L. Y. Wang, Y. M. Xie, X. J. Han, and A. P. Lu, "Development of clinical practice guidelines in 11 common diseases with Chinese medicine interventions in China," Chinese Journal Integrative Medicine, vol. 18, no. 2, pp. 112-119, 2012.

[5] C. H. Yeh, C. K. Arnold, Y. Chen, and J. N. Lai, "Suan Zao Ren Tang as an original treatment for sleep difficulty in climacteric women: a prospective clinical observation," Evidence-based Complementary and Alternative Medicine, vol. 2011, Article ID 673813, 8 pages, 2011.

[6] H. C. Wu, Y. H. Chen, J. N. Lai, J. S. Hwang, and J. D. Wang, "Improving sleep quality in climacteric women with insomnia: a randomized, head-to-head trial between Jia-Wei-Shiau-Yau San (JWSYS) and Suan-Zao-Ren Tang (SZRT)," European Journal of Integrative Medicine, vol. 3, no. 3, pp. e143-e151, 2011.
[7] L. C. Chang, N. Huang, Y. J. Chou, C. H. Lee, F. Y. Kao, and Y. Huang, "Utilization patterns of Chinese medicine and Western medicine under the National Health Insurance Program in Taiwan, a population-based study from 1997 to 2003," BMC Health Services Research, vol. 8, p. 170, 2008.

[8] S. C. Hsieh, J. N. Lai, C. F. Lee, F. C. Hu, W. L. Tseng, and J. D. Wang, "The prescribing of Chinese herbal products in Taiwan: a cross-sectional analysis of the national health insurance reimbursement database," Pharmacoepidemiology and Drug Safety, vol. 17, no. 6, pp. 609-619, 2008.

[9] Y. C. Lee, Y. T. Huang, Y. W. Tsai et al., “The impact of universal National Health Insurance on population health: the experience of Taiwan," BMC Health Services Research, vol. 10, p. 225, 2010.

[10] N. H. R. Institutes, "National Health Insurance Research database," 2013, http://www.nhri.org.tw/nhird/date_01.html\#_ edn1.

[11] C. f. D. a. Prevention, "International Classification of Diseases, Ninth Revision (ICD-9)," http://www.cdc.gov/nchs/icd/icd9 .htm.

[12] Department of Chinese Medicine and Pharmacy, Ministry of Health and Welfare, Executive Yuan, Taiwan, "List of 100 unified formulae," 2013, http://www.mohw.gov.tw/CHT/DOCMAP/ DM1_Paspx?f_list_no=758\&fod_list_no=4250\&doc_no $=32620$.

[13] M. Warner, L. H. Chen, and D. M. Makuc, "Increase in fatal poisonings involving opioid analgesics in the United States, 1999-2006," NCHS Data Brief, vol. 81, no. 22, pp. 1-8, 2009.

[14] Y. H. Yang, J. N. Lai, C. H. Lee, J. D. Wang, and P. C. Chen, "Increased risk of hospitalization related to motor vehicle accidents among people taking zolpidem: a case-crossover study," Journal of Epidemiology, vol. 21, no. 1, pp. 37-43, 2011.

[15] M. Calem, J. Bisla, A. Begum et al., "Increased prevalence of insomnia and changes in hypnotics use in England over 15 years: analysis of the 1993, 2000, and 2007 national psychiatric morbidity surveys," Sleep, vol. 35, no. 3, pp. 377-384, 2012.

[16] M. Angell and J. P. Kassirer, "Alternative medicine-the risks of untested and unregulated remedies," New England Journal of Medicine, vol. 339, no. 12, pp. 839-841, 1998.

[17] S. Hwang, F. Chen, M. Jong et al., "Prescriptions of Chinese herbal medicines for insomnia in Taiwan during 2002," Evidence-based Complementary and Alternative Medicine, vol. 2011, Article ID 236341, 9 pages, 2011.

[18] H. Hachul, T. K. Garcia, A. L. Maciel, F. Yagihara, S. Tufik, and L. Bittencourt, "Acupuncture improves sleep in postmenopause in a randomized, double-blind, placebo-controlled study," Climacteric, vol. 16, no. 1, pp. 36-40, 2012.

[19] J. Tu, W. Chung, C. Yang, and D. Tzeng, "A comparison between acupuncture versus zolpidem in the treatment of primary insomnia," Asian Journal of Psychiatry, vol. 5, no. 3, pp. 231-235, 2012. 


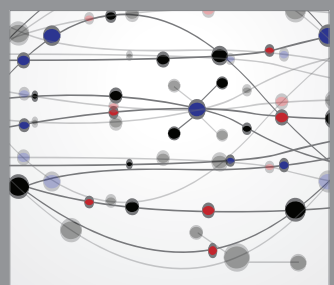

The Scientific World Journal
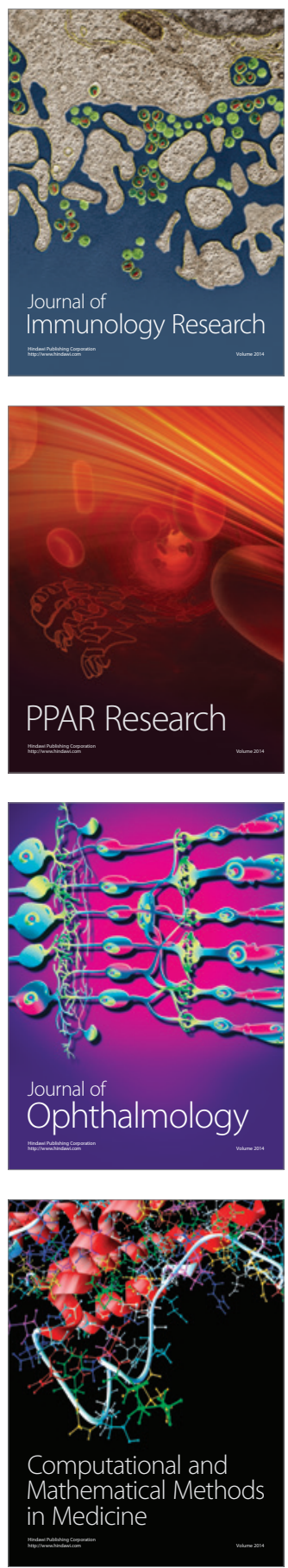

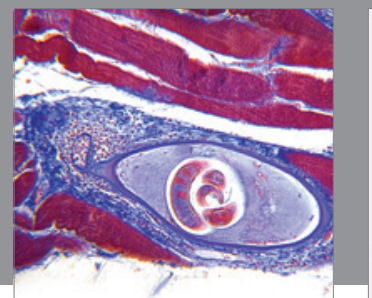

Gastroenterology

Research and Practice
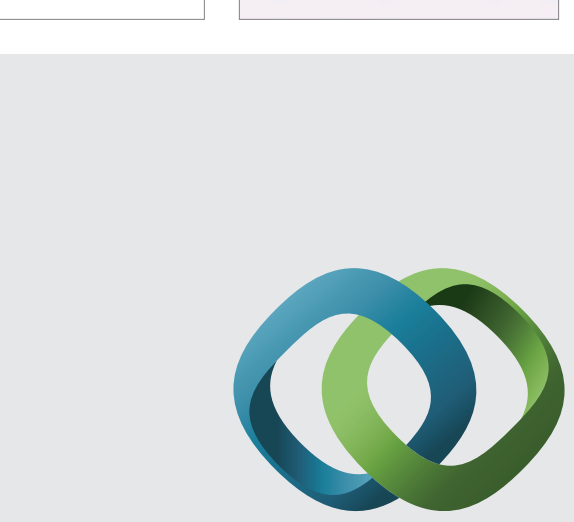

\section{Hindawi}

Submit your manuscripts at

http://www.hindawi.com
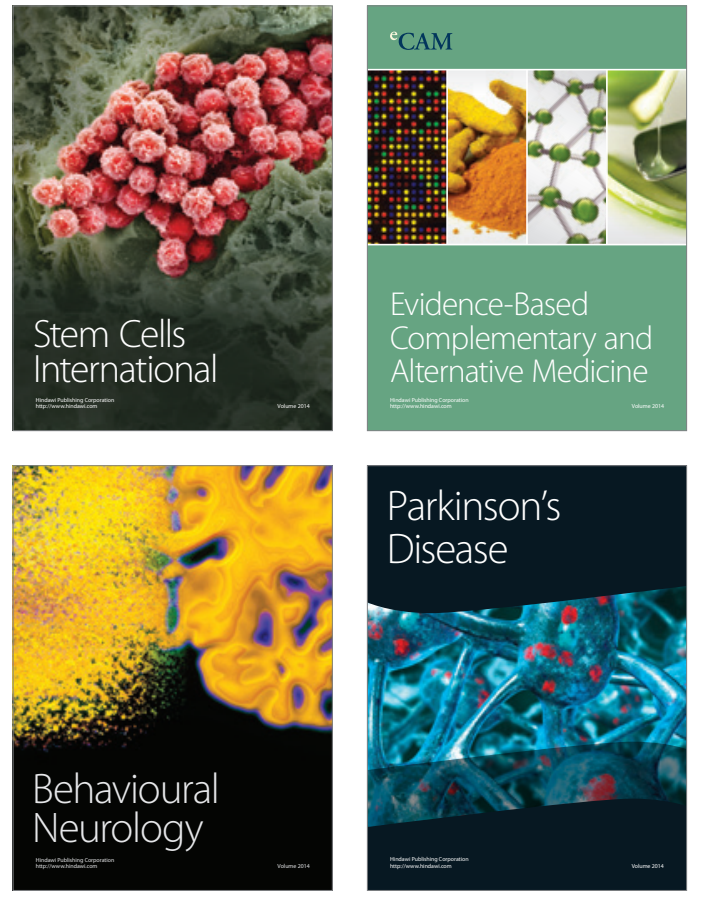
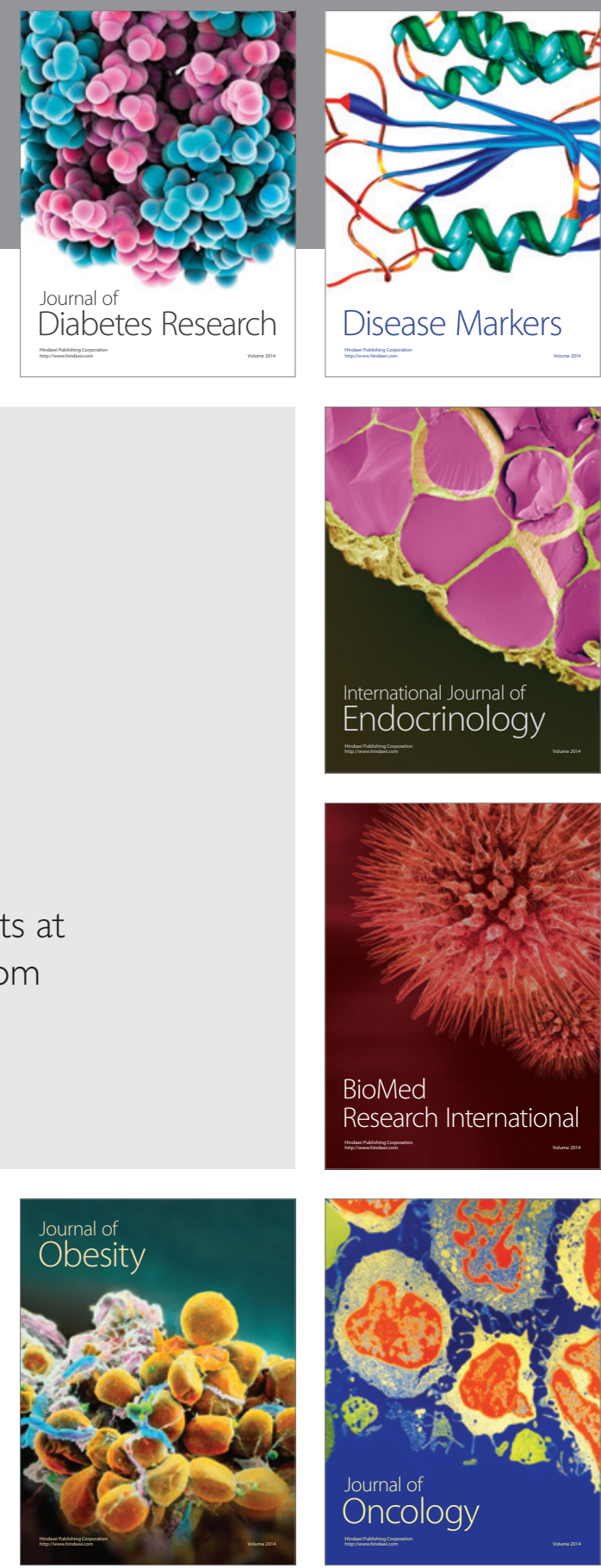

Disease Markers
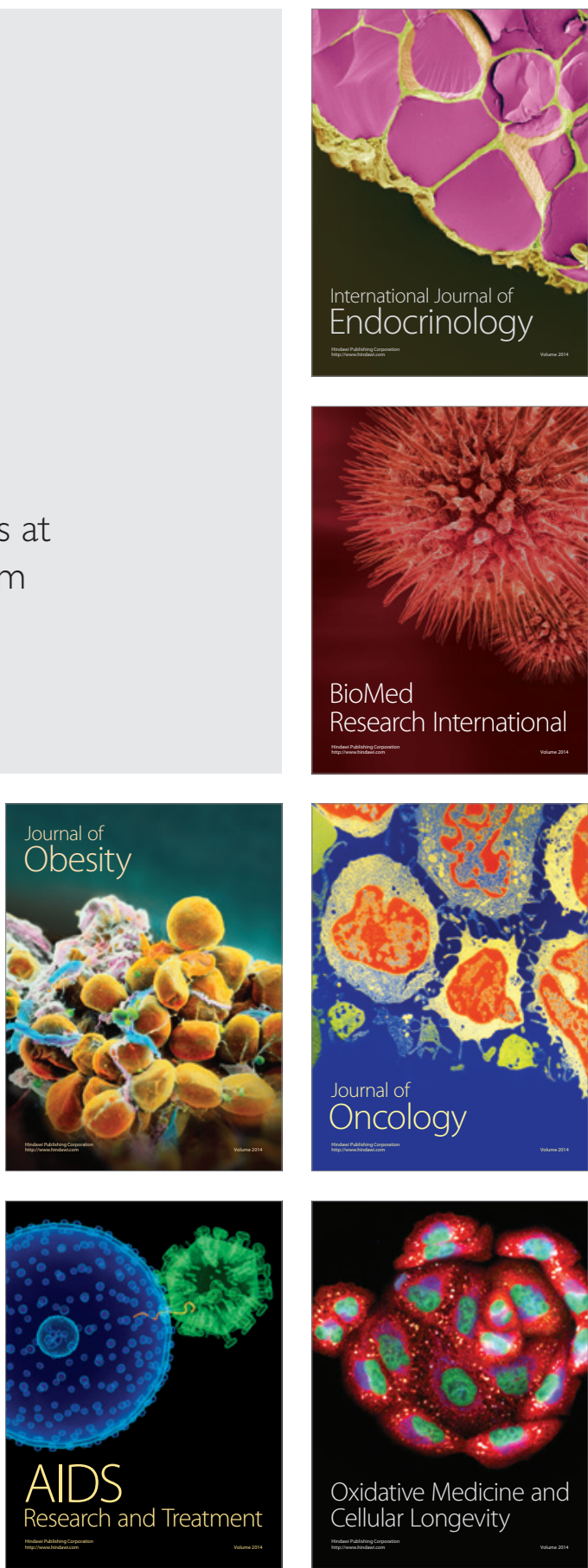